\title{
Lentiviral-mediated overexpression of homeobox A4 by human umbilical cord mesenchymal stem cells repairs full-thickness skin defects
}

\author{
LING HE $^{1 *}$, HUAI-JUN TU ${ }^{2 *}$, WEN-FENG HE ${ }^{1}$, LING-LING GUO ${ }^{1}$, \\ SONG-XIA YU ${ }^{1}$, JIE LI ${ }^{1}$, QIONG WU ${ }^{1}$ and JIAN LI ${ }^{1}$ \\ ${ }^{1}$ Key Laboratory of Hematology and ${ }^{2}$ Department of Neurology, The Second Affiliated Hospital of Nanchang University, \\ Nanchang, Jiangxi 330006, P.R. China
}

Received June 26, 2013; Accepted October 1, 2014

DOI: $10.3892 / \mathrm{mmr} .2015 .3208$

\begin{abstract}
A number of types of stem cells have been shown to be effective in wound repair. In the present study the effect of homeobox A4 (HOXA4) overexpression by human umbilical cord mesenchymal stem cells (hUMSCs) on full-thickness skin repair was evaluated. Isolated hUMSCs were transfected with a lentivirus expressing HOXA4 and cultured for 21 days. Expression of the epidermal cell-specific markers, cytokeratins 14 and 18, was detected by immunohistochemistry and flow cytometry. Full-thickness skin defects $(1.5 \mathrm{~cm}$ x $1.5 \mathrm{~cm})$ were made on the backs of 45 nude mice, which were randomly divided into the following three treatment groups: Collagen membrane with lenti-HOXA4 hUMSC seed cells; collagen membrane with lentivirus expressing green fluorescent protein; and collagen membrane alone. On days 7, 14 and 21 following transplantation, tissue samples were harvested and examined by histology and western blot analysis. Flow cytometry showed that the transfection efficiency was $95.41 \%$ at a multiplicity of infection of 100, and that the lenti-HOXA4 hUMSCs differentiated into epidermal cells, expressing cytokeratins 14 and 18. In addition, re-epithelialization of wounds treated with lenti-HOXA4 hUMSCs was significantly greater than that in the control groups in the first week. By week three the epidermis was significantly thicker in the lenti-HOXA4 group than the control groups. Thus, transplantation of hUMSCs modified with Ad-HOXA4 promoted wound healing.
\end{abstract}

Correspondence to: Dr Jian Li, Key Laboratory of Hematology, The Second Affiliated Hospital of Nanchang University, 1 Minde Road, Nanchang, Jiangxi 330006, P.R. China

E-mail: thj127900@163.com

${ }^{*}$ Contributed equally

Key words: human umbilical cord mesenchymal stem cell, epidermal-like cells, homeobox A4, transfection, wound healing

\section{Introduction}

Advances in understanding of the molecular mechanisms involved in wound repair have demonstrated a role for distant stem cells in supporting wound healing $(1,2)$. Human umbilical cord mesenchymal stem cells (hUMSCs) may represent an important seed cell for tissue engineering due to their capacity for self-renewal and multilineage differentiation $(3,4)$. Stem cells may also represent an effective vehicle for gene delivery $(5,6)$.

The homeobox $(H O X)$ family of transcription factors influence bone marrow-derived cell differentiation, migration and adhesion to injured tissue (7). In multicellular organisms, HOX genes regulate body morphology formation, and the differentiation and embryonic development of the axial skeleton, gastrointestinal tract, urogenital tube, external genital duct, central nervous system, hematopoietic system, and the limbs and skin $(8,9)$. In adults, HOX genes are involved in maintaining positional identity, tissue homeostasis and remodeling in normal tissue and during wound healing $(7,10)$. HOXA4 gene expression has been observed in keratinocytes, fibroblasts and mesenchymal stem cells in embryonic and adult skin, as well as hair follicles (11). In addition, HOXA4 regulates cell chemotaxis, migration, proliferation and differentiation during the reconstruction and healing of damaged tissue (5).

The present study analyzed whether HOXA4 expression by hUMSCs enhanced the differentiation of these cells into epidermal-like cells in order to promote skin repair. The aim was to provide a basis for the development of novel combined gene and stem cell-based therapies for wound repair.

\section{Materials and methods}

hUMSC isolation, culture and phenotype analysis. This study was approved by the institutional review board of the Second Affiliated Hospital of Nancheng University (Nanchang, China) in compliance with the Declaration of Helsinki. Written informed consent was obtained from the patients' families, for removal and storage of a fresh human umbilical cord from a single infant in Hank's balanced salt solution (Sigma-Aldrich, St Louis, MO, USA) for $24 \mathrm{~h}$ prior to the isolation of hUMSCs 
as previously described (12). Cells were cultured in Dulbecco's modified Eagle's medium (DMEM; Gibco Life Technologies, Carlsbad, CA, USA) supplemented with $10 \%$ fetal bovine serum (FBS; GE Healthcare Life Sciences, Logan, UT, USA) and glucose $(4.5 \mathrm{~g} / \mathrm{l})$ in $5 \% \mathrm{CO}_{2}$ at $37^{\circ} \mathrm{C}$ for 14 days.

The identity of the isolated hUMSCs was confirmed using a FACSCalibur Flow Cytometer (BD Biosciences, San Diego, CA, USA) and fluorescein isothiocyanate (FITC)-conjugated antibodies against CD105, CD54, CD33 and CD29, and phycoerythrin (PE)-conjugated antibodies against CD45, CD166, CD34 and CD13 (all from BD Biosciences). FITC- and PE-conjugated human IgG1 were used as isotype-matched controls to set gates.

hUMSC infection and differentiation. Cells in the third passage were infected with a lentiviral vector containing either green fluorescent protein alone (lenti-GFP) or GFP and HOXA4 (lenti-HOXA4, Invitrogen Life Technologies, Grand Island, NY, USA) overnight at a multiplicity of infection (MOI) of 60 plaque-forming units (PFU)/cell. hUMSCs were incubated in DMEM/F-12 media supplemented with $0.5 \mu \mathrm{M}$ dexamethasone (Sigma-Aldrich) and $10 \mathrm{ng} / \mathrm{ml}$ epidermal growth factor (PeproTech, Inc., Rocky Hill, NJ, USA) for 2 weeks. The efficiency of transduction was determined by analysis of GFP expression using fluorescence microscopy (TE-2000, Nikon Corporation, Tokyo, Japan), by calculating the ratio of GFP-positive cells (green fluorescence) to the total number of cells.

Flow cytometry. Cells were suspended in DMEM $\left(5 \times 10^{6} / \mathrm{ml}\right)$, permeabilized in $0.1 \%$ Triton X-100 (Sigma-Aldrich), and incubated with $150 \mu \mathrm{l}$ mouse anti-human cytokeratin 14 and 18 antibodies followed by FITC-conjugated anti-mouse antibodies (10 mg/ml; all from BD Biosciences). Cells were washed twice with phosphate-buffered saline (PBS), centrifuged at $600 \mathrm{x} \mathrm{g}$ for $5 \mathrm{~min}$, and fixed in $1.5 \mathrm{ml}$ of $4 \%$ paraformaldehyde. Control samples were incubated with PBS in place of a primary antibody or an isotype-matched control IgG. Cell labeling was evaluated in $1 \times 10^{6}$ cells using a FACScan (BD Biosciences).

Reverse transcription-polymerase chain reaction (RT-PCR). Total RNA was isolated using TRIzol ${ }^{\circledR}$ reagent (Invitrogen Life Technologies), and $1 \mu \mathrm{g}$ was used for cDNA synthesis using the Transcriptor Universal cDNA Master (Roche Diagnostics, Indianapolis, IN, USA). cDNA was obtained by reverse transcription according to the manufacturer's instructions (Tiangen Biotech Co., Ltd., Beijing, China). The PCR reaction was conducted using $1 \mu \mathrm{l}$ cDNA, $5 \mu \mathrm{l}$ of 10X PCR buffer, (Takara Bio, Inc., Otsu, Japan) $1.5 \mu \mathrm{l}$ of $2 \mathrm{mM}$ magnesium chloride, $1 \mu \mathrm{l}$ specific HOXA4 primers (20 pM per primer), $1 \mu \mathrm{l}$ of $10 \mathrm{mM}$ dNTP mix (Takara Bio, Inc.), and $1 \mu \mathrm{l}$ Taq DNA polymerase $(5 \mathrm{U} / \mathrm{ml}$; Takara Bio, Inc.) in a total volume of $0.5 \mu 1$. The HOXA4 primer sequences were as follows: Forward: 5'-CGCGCTAGCATGACCATGAGCTCGTTTTTG-3' and reverse: 5'-GCGCGATCGTACTGGTAC TCGAGCAAAAAC-3'. Samples were amplified in a thermocycler (Applied Biosystems Life Technologies, Foster City, CA, USA) under the following conditions: $95^{\circ} \mathrm{C}$ for $2 \mathrm{~min}$; $95^{\circ} \mathrm{C}$ for $30 \mathrm{sec}, 55^{\circ} \mathrm{C}$ for $54 \mathrm{sec}$ and $72^{\circ} \mathrm{C}$ for $60 \mathrm{sec}$ for
33 cycles; and $72^{\circ} \mathrm{C}$ for $5 \mathrm{~min}$. The PCR products were separated with an agarose gel and stained with ethidium bromide (Sigma-Aldrich).

Immunocytochemistry. Cells were fixed in $4 \%$ paraformaldehyde, permeabilized and blocked with $0.4 \%$ Triton X-100 and $5 \%$ bovine serum albumin (Sigma-Aldrich) in PBS, and incubated with human-cytokeratin 14 and 18 antibodies (BD Biosciences). Following incubation with biotinylated goat anti-rabbit IgG, staining was visualized using an avidin biotin complex (ABC)-peroxidase kit (Sigma-Aldrich), 3,3-diaminobenzidine tetrahydrochloride and counterstained with hematoxylin. For the negative control, the primary antibody was omitted. The slides were observed under an Olympus microscope (BX51, Olympus Corporation, Tokyo, Japan), in order to calculate the ratio of positive cells (brown fluorescence) to the total number of cells.

In vivo model of full-thickness skin defects. Collagen was prepared from the tails of Sprague-Dawley rats as previously described (13). All animal procedures used approved protocols in accordance with the regulations of the Animal Care and Experiment Committee of the Nanchang University School of Medicine. Forty-five nude mice were purchased from Shanghai Experimental Animal Laboratory (Shanghai, China). Following intraperitoneal administration of ketamine (10 mg/100 g; Sigma-Aldrich), full-thickness skin defects $\left(1.5 \times 1.5 \mathrm{~cm}^{2}\right)$ were made on the backs of all nude mice, which were randomly divided into the following groups, with 15 mice in each group: Collagen membrane with $1 \times 10^{6}$ lenti-HOXA4 hUMSCs; collagen membrane with $1 \times 10^{6}$ lenti-GFP hUMSCs and collagen membrane alone. Cells were seeded evenly on the collagen membrane, which was then directly applied to the wound as previously described (14). At day 21, the mice were sacrificed by cervical dislocation following anesthesia with IP ketamine $(100 \mathrm{mg} / \mathrm{kg}$ ) and xylazine ( $5 \mathrm{mg} / \mathrm{kg}$; Sigma-Aldrich), and wound tissue samples were harvested. One section of tissue was fixed with $10 \%$ formalin, cut into $3-\mu \mathrm{m}$ sections, stained with hematoxylin and eosin, and observed with a light microscope (BX51, Olympus Corporation). The remaining piece was processed for western blot analysis.

Western blot analysis. Total protein was extracted using a triplex lysis buffer (Roche Diagnostics, Basel, Switzerland). Total protein $(20 \mu \mathrm{g})$ was separated on a $10 \%$ SDS polyacrylamide gel and transferred to a polyvinylidene fluoride membrane (EMD Millipore, Billerca, MA, USA). The membrane was blocked with non-fat dry milk and incubated with mouse monoclonal anti-HOXA4 antibody (1:100) or mouse monoclonal anti-actin antibody (1:500) (Santa Cruz Biotechnology, Inc., Dallas, TX, USA) at $4^{\circ} \mathrm{C}$ overnight, followed by incubation with monoclonal rabbit anti-mouse horseradish peroxidase (HRP)-conjugated secondary antibody (1:5,000; Santa Cruz Biotechnology, Inc.) at room temperature for 1-2 h. Color was developed using an enhanced chemiluminescence kit (Thermo Fisher, Scientific, Waltham, MA, USA). The relative HOXA4 protein expression was determined as the gray value of HOXA4 band/gray value of actin band using a gel imaging analysis system (Syngene, Frederick, MD, USA). 
$\mathbf{A}$
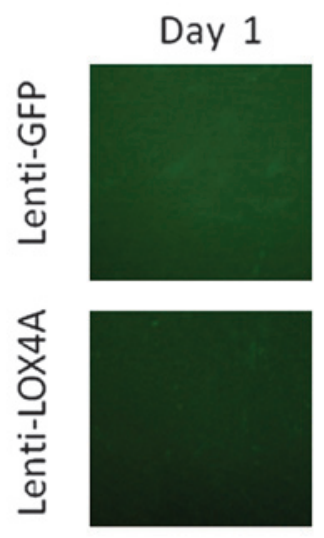

Day 3
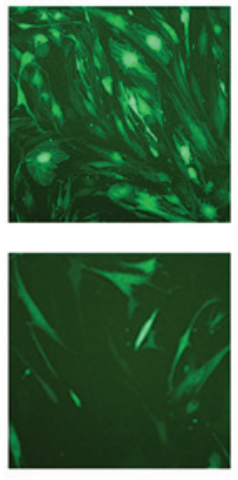

Day 7
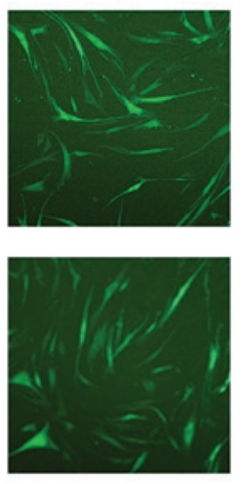

Day 14
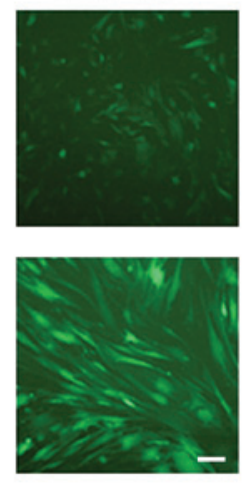

B

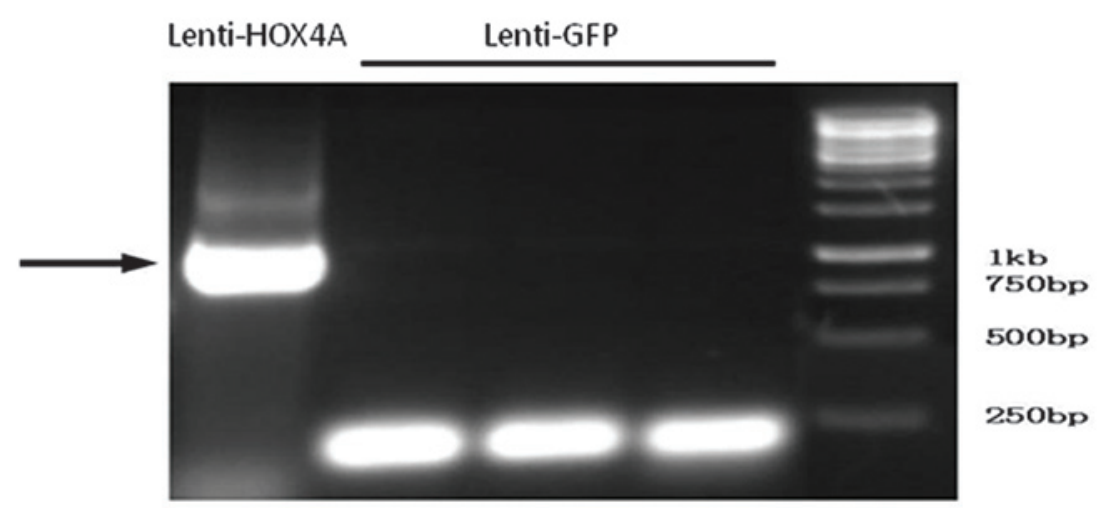

Figure 1. HOXA4 gene transduction of hUMSCs. (A) Fluorescence microscopy of GFP expression at 1,3, 7 and 14 days following infection with lenti-GFP (top panels) or lenti-HOXA4 (bottom panels). Magnification, x100; scale bar, $25 \mu \mathrm{m}$. (B) Reverse transcription-polymerase chain reaction analysis of $H O X A 4$ and GFP expression in hUMSCs 7 days following infection with lenti-GFP or lenti-HOXA4. HOXA4, homeobox A4, hUMSCs, human umbilical cord mesenchymal stem cells; lenti-GRP, lentivirus expressing green fluorescent protein; lenti-HOXA4, lentivirus expressing HOXA4.

Statistical analysis. Continuous variables are presented as the mean \pm standard deviation. For comparisons of three treatment groups, one-way analysis of variance was undertaken. When a significant difference between groups was apparent, multiple comparisons were performed using the Bonferroni procedure with type I error adjustment. All statistical assessments were two-sided and $\mathrm{P}<0.05$ was considered to indicate a statistically significant difference. SAS software package, version 9.2 (SAS Institute, Inc., Cary, NC, USA) was used for statistical analysis.

\section{Results}

Identity of the isolated hUMSCs. The percentage of cells positive for the markers investigated was as follows: CD13,

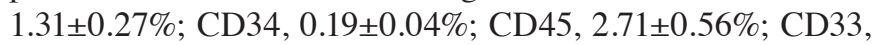

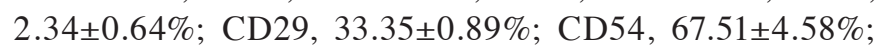

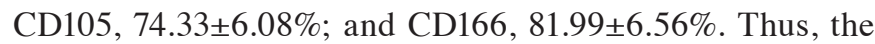
isolated cells were confirmed as hUMSCs.

HOXA4 gene transduction of hUMSCs. The efficiency of lenti-HOXA4 gene transduction was determined in each group simultaneously by evaluating the percentages of GFP-positive hUMSCs at days 3, 7 and 14, which were 90.3, 81.6 and 70.4\%, respectively (Fig. 1A). HOXA4 gene expression was analyzed using RT-PCR following transduction and culture in a normal medium. At day 7, hUMSCs transduced with lenti-HOXA4 expressed higher levels of HOXA4 than those cells infected with lenti-GFP (Fig. 1B).

Increased epidermal cell marker expression in lenti-HOXA4 hUMSCs. At 14 days post-lenti-HOXA4 infection, expression of cytokeratin 14 and 18 was increased compared with that in the control groups (Fig. 2A). The proportion of CK14-positive cells in the hUMSCs, lenti-GFP hUMSCs and lenti-HOXA4 hUMSCs groups was $1.22 \pm 0.13,1.31 \pm 0.24$ and $32.98 \pm 5.12 \%$, respectively. The proportion of CK18-positive cells was $1.22 \pm 0.13,1.3 \pm 0.14$ and $45.13 \pm 4.5 \%$ in the hUMSCs, lenti-GFP hUMSCs and lenti-HOXA4 hUMSCs, respectively. These results were confirmed using flow cytometric analysis. In hUMSCs, CK14 and CK18 expression was observed in 1.58 and $5.17 \%$ of the cells, respectively; whereas these markers were detected in 32.84 and $41.14 \%$, respectively, of cells transduced with lenti-HOXA4 (Fig. 2B).

In vivo epidermal regeneration with HOXA4-expressing hUMSCs. Mice with full-thickness skin defects were treated with collagen membrane alone, or evenly seeded with lenti-GFP hUMSCs or lenti-HOXA4 hUMSCs, and histological analysis was undertaken at day 21 post-injury (Fig. 3), at which time macroscopic evidence of wound healing was 

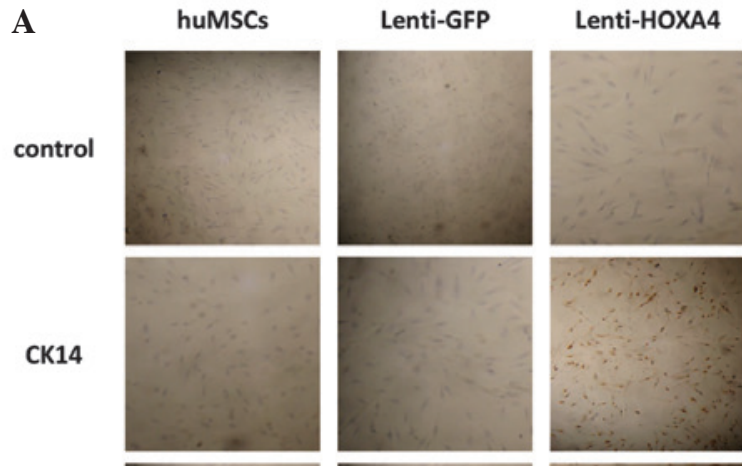

CK18
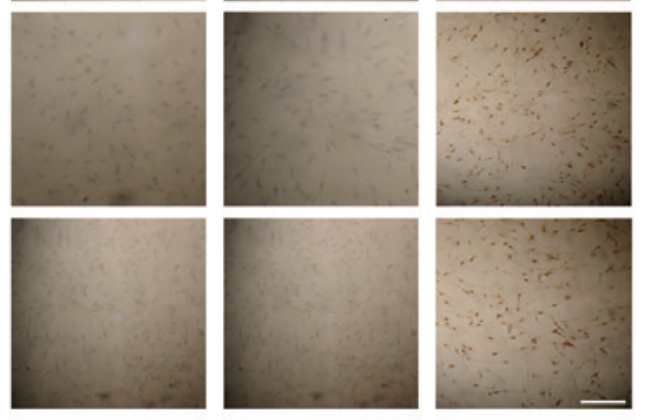

B
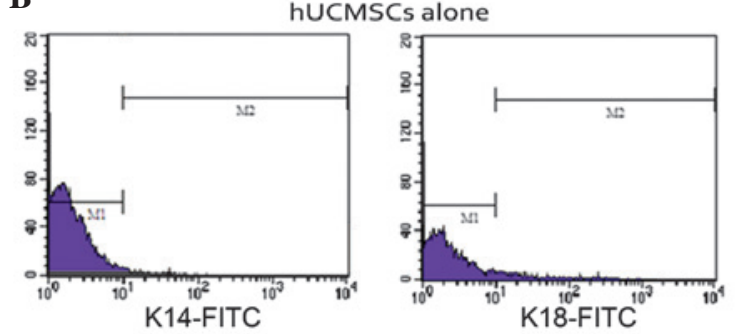

Lenti-HOX4AhUCMSCS
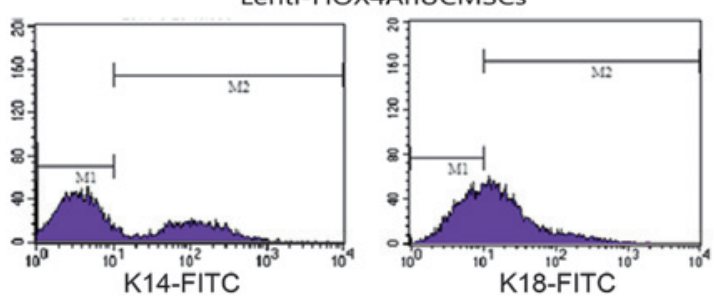

Isotype-matched control

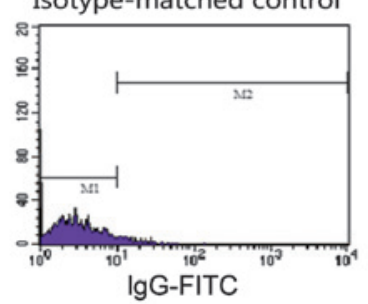

Figure 2. Expression of epidermal cell marker expression (cytokeratin 14 and 18) in lenti-HOXA4 hUMSCs, lenti-GFP hUMSCs and control hUMSCs. (A) Immunocytochemical analysis at day 14 post-lenti-HOXA4 infection (magnification, $\mathrm{x} 100$; scale bar, $100 \mu \mathrm{m}$ ). (B) Flow cytometric analysis. Analysis of isotype-matched IgG-FITC served as a control. Lenti-HOXA4, lentivirus expressing homeobox A4; lenti-GFP, lentivirus expressing green fluorescent protein; hUMSCs, human umbilical cord mesenchymal stem cells, FITC, fluorescein isothiocyanate.

also evident. In the collagen membrane control group, the epidermis did not heal, or scar tissue was formed (Fig. 3A; arrow). Mice treated with lenti-HOXA4 hUMSCs had a thicker epidermis with more organized cell layers compared with that of the lenti-GFP hUMSC group (Fig. 3B and C, respectively). Furthermore, western blot analysis of the tissues indicated persistent HOXA4 expression in the wounds of mice treated with lenti-HOXA4 hUMSCs (Fig. 4A, lane 1), which was
A

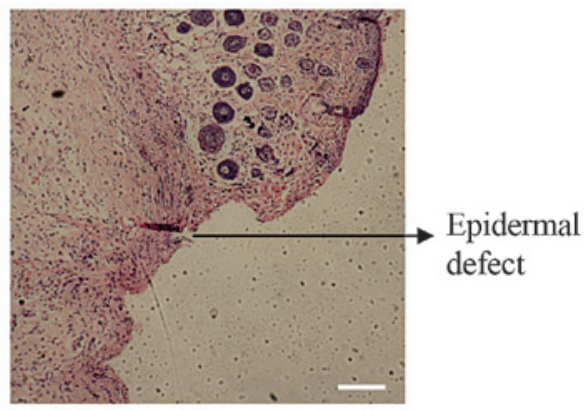

B

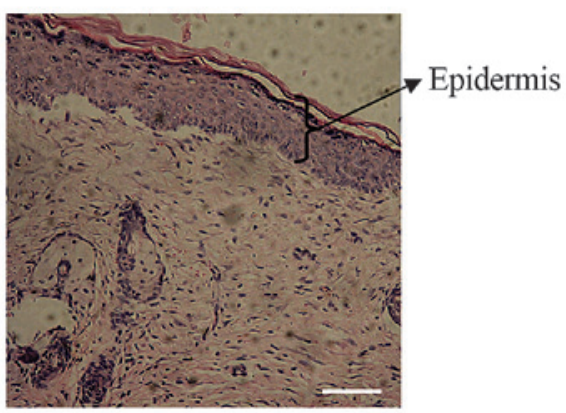

C

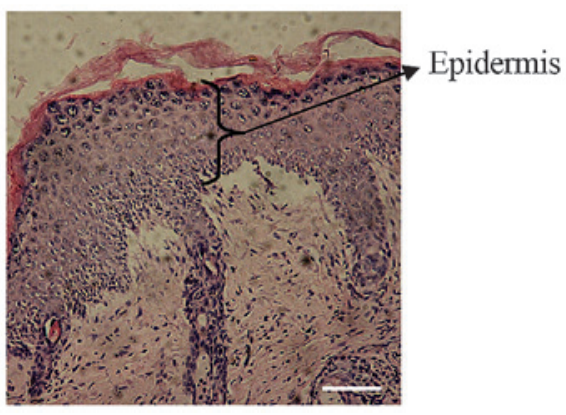

Figure 3. In vivo epidermal regeneration following xenografts with HOXA4-expressing hUMSCs. Full-thickness skin defects were treated with (A) a collagen membrane alone, or a collagen membrane evenly seeded with either (B) lenti-GFP hUMSCs or (C) lenti-HOXA4 hUMSCs. At day 21, histological analysis of the wounds was undertaken following hematoxylin and eosin staining. In the collagen membrane control group, the skin was not fully healed, and new epidermis was not formed (arrow; magnification, x40; scale bar, $200 \mu \mathrm{m}$ ). In the lenti-GFP hUMSC and lenti-HOXA4 hUMSC groups, the epidermis (arrows) was formed (magnification, x100; scale bar, $100 \mu \mathrm{m}$ ). However, the epidermis was thicker and the cell layers more organized in the wounds treated with $H O X A 4$-expressing hUMSCs than in the control group. Lenti-HOXA4, lentivirus expressing homeobox A4; hUMSCs, human umbilical cord mesenchymal stem cells; lenti-GFP, lentivirus expressing green fluorescent protein.

significantly higher than that in the hUMSCs and lenti-GFP hUMSC groups $(\mathrm{P}<0.001$, Fig. 4B). Expression of cytokeratins 14 and 18 was also detected in mice treated with lenti-GFP hUMSCs and lenti-HOX4A (Fig. 4C, lane 2); however, the expression of each of these cytokeratins was significantly higher in the lenti-HOXA4 hUMSC group than in the lenti-GFP hUMSC group ( $\mathrm{P} \leq 0.003$ and $\mathrm{P} \leq 0.001$, respectively; Fig. 4C and 4D).

\section{Discussion}

The present study sought to determine whether HOXA4 expression in hUMSCs enhanced their differentiation into 
$\mathbf{A}$

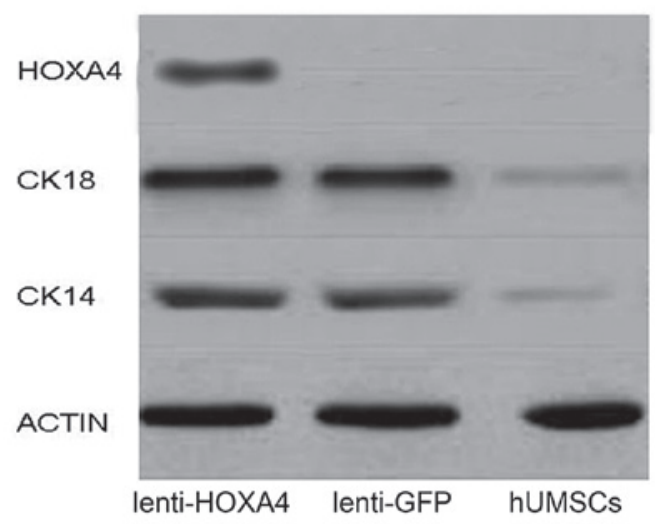

C

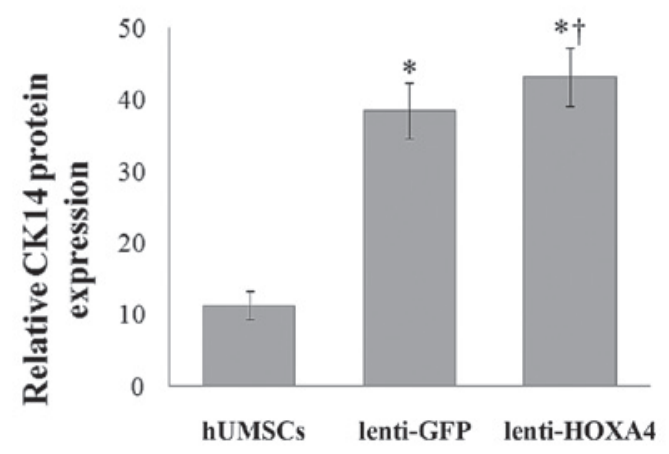

$\mathbf{B}$

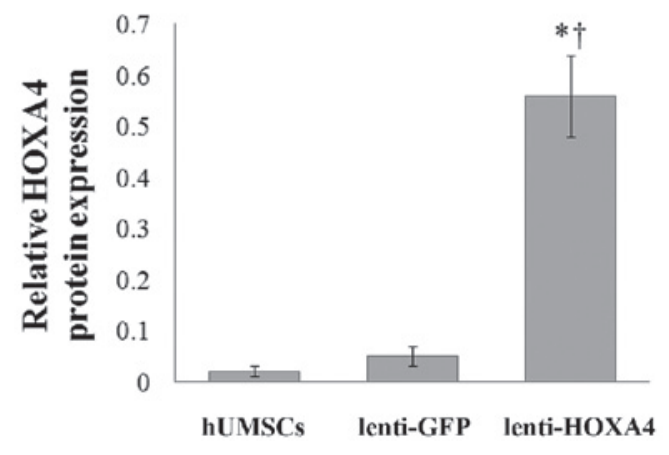

D

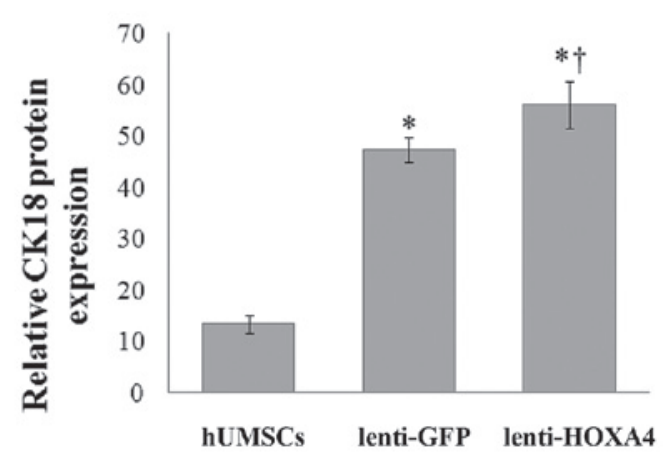

Figure 4. HOXA4, CK14 and CK18 protein expression during epidermal regeneration following xenografts with HOXA4-expressing hUMSCs in vivo. (A) Representative western blot of HOXA4, CK14 and CK18 protein expression. Quantitative analysis of (B) HOXA4, (C) CK14 and (D) CK18 protein expression in the hUMSCs, lenti-GFP hUMSCs, and lenti-HOXA4 hU MSCs groups ( $\mathrm{n}=15$ per group). ${ }^{*} \mathrm{P}<0.05$, compared with the hUMSC control group and ${ }^{\dagger} \mathrm{P}<0.05$, compared with the lenti-GFP hUMSC group. HOXA4, homeobox A4; hUMSCs, human umbilical cord mesenchymal stem cells; lenti-GFP, lentivirus expressing green fluorescent protein; lenti-HOXA4, lentivirus expressing HOXA4; CK, cytokeratin.

epidermal-like cells and therefore facilitated the process of skin repair. In lenti-HOXA4 hUMSCs, increased cytokeratin 14 and 18 expression was observed along with enhanced repair of full-thickness skin defects.

Since embryonic MSCs are able to differentiate into epidermal cells in vitro and in vivo (15), the application of these cells in acute and chronic wound therapy has been investigated. Studies have shown that following transplantation via tail vein injection, hUMSCs migrate to the skin and differentiate into epithelial cells $(16,17)$. In addition, an artificial skin, consisting of epidermal stem cells and fibroblasts on a collagen lattice, was shown to promote wound healing in mice (18). Besides their regenerative capacity, the use of MSCs as vehicles for gene delivery has been explored $(5,6)$. Improved wound healing was reported following the administration of MSCs expressing various growth factors and cytokines (19-21). In the present study, persistent expression of HOXA4 in hUMSCs was observed at 21 days following transduction, suggesting that these cells are also capable of carrying foreign genes, thus potentially enhancing cell therapy.

The HOXA4 gene is expressed in skin, amongst other tissues $(22,23)$ and is known to regulate cell differentiation during the reconstruction and healing of damaged tissue (5). We therefore hypothesized that its expression by hUMSCs may enhance their capacity to differentiate into epidermal cells. Following incubation with epidermal-inducing agents, HOXA4-expressing hUMSCs differentiated into epidermal-like cells and were demonstrated to express cytokeratins 14 and 18 . Further studies may thus directly assess the effects of HOXA4 expression on hUMSC differentiation into keratinocytes. Furthermore, recent data have indicated that Mospdl gene expression may regulate mesenchymal to epithelial transition $(13,18)$; therefore, future studies may be conducted in order to assess its value in promoting differentiation.

In the current study, wounds treated with lenti-HOXA4 hUMSCs appeared to have a thicker epidermis on day 21 following injury. Thus, lenti-HOXA4 hUMSCs may improve wound healing and reduce scar formation. However, the mechanism underlying these effects remains unknown. The expression of HOXA4 may, for example, increase the expression of Cyclin D1 and Bcl-2, or it may reduce the expression of P21WAF/CIPI, thereby promoting cell proliferation and inhibiting apoptosis (24). This group intends to investigate these possible mechanisms in future studies.

The present study is limited in that the re-epithelialized epidermis was not evaluated for the presence of hUMSC-derived keratinocytes. In addition, the extent of wound healing was only assessed on day 21; therefore, differences at earlier time points may have been missed. Furthermore, changes in the epidermal thickness were not quantified. Finally, although HOXA4 mRNA 
expression was determined following transduction of the hUMSCs, its protein expression levels were not assessed.

In conclusion, lenti-HOXA4 hUMSCs enhanced wound repair and may represent a novel therapeutic approach for burns and chronic wounds. Further studies are required to elucidate the molecular mechanisms governing this promotion of wound healing.

\section{Acknowledgements}

The authors would like to thank Professor Yucheng Dai (Institution of Hematology, the Second Affiliated Hospital of Nanchang University) for his assistance. This study was supported by the National Natural Science Foundation of China (grant no. 30760261), the Natural Science Foundation of Jiangxi Province (grant no. 0540084) and the Project of Cultivating Academic and Technical Leaders for Major Disciplines in Jiangxi province (grant no. 2014BCB22009).

\section{References}

1. Ballas CB, Zielske SP and Gerson SL: Adult bone marrow stem cells for cell and gene therapies: implications for greater use. J Cell Biochem 38 (Suppl): 20-28, 2002.

2. Fathke C, Wilson L, Hutter J, Kapoor V, Smith A, Hocking A and Isik F: Contribution of bone marrow-derived cells to skin: collagen deposition and wound repair. Stem Cells 22: 812-822, 2004.

3. Chamberlain G, Fox J, Ashton B and Middleton J: Concise review: mesenchymal stem cells: their phenotype, differentiation capacity, immunological features, and potential for homing. Stem Cells 25: 2739-2749, 2007.

4. Pittenger MF, Mackay AM, Beck SC, Jaiswal RK, Douglas R, Mosca JD, Moorman MA, Simonetti DW, Craig S and Marshak DR: Multilineage potential of adult human mesenchymal stem cells. Science 284: 143-147, 1999.

5. Branski LK, Gauglitz GG, Herndon DN and Jeschke MG: A review of gene and stem cell therapy in cutaneous wound healing. Burns 35: 171-180, 2009.

6. Gauglitz GG and Jeschke MG: Combined gene and stem cell therapy for cutaneous wound healing. Mol Pharm 8: 1471-1479, 2011.

7. Mahdipour E and Mace KA: Hox transcription factor regulation of adult bone-marrow-derived cell behaviour during tissue repair and regeneration. Expert Opin Biol Ther 11: 1079-1090, 2011.

8. Akiyama M, Smith LT and Holbrook KA: Growth factor and growth factor receptor localization in the hair follicle bulge and associated tissue in human fetus. J Invest Dermatol 106: 391-396, 1996.
9. Favier B and Dollé P: Developmental functions of mammalian Hox genes. Mol Hum Reprod 3: 115-131, 1997.

10. Wang KC, Helms JA and Chang HY: Regeneration, repair and remembering identity: the three Rs of Hox gene expression. Trends Cell Biol 19: 268-275, 2009.

11. Stelnicki EJ, Kömüves LG, Kwong AO, Holmes D, Klein P, Rozenfeld S, Lawrence HJ, Adzick NS, Harrison M and Largman C: HOX homeobox genes exhibit spatial and temporal changes in expression during human skin development. J Invest Dermatol 110: 110-115, 1998 .

12. Hsieh JY, Wang HW, Chang SJ, Liao KH, Lee IH, Lin WS, Wu CH, Lin WY and Cheng SM: Mesenchymal stem cells from human umbilical cord express preferentially secreted factors related to neuroprotection, neurogenesis, and angiogenesis. PLoS One 8: e72604, 2013.

13. Hu K, Dai Y and Yuan J: An experimental study on the preparation of artificial dermis. J Xian Jiaotong Univ (Medical Sciences) 27: 117-119, 131, 2006 (In Chinese).

14. Hu K, Dai Y, Hu Q, Li J, Yuan J, Li J and Wu Q: An experimental study on the repair of full skin loss of nude mice with composite graft of epidermal stem cells. Burns 32: 416-422, 2006.

15. Wu M, Yang L, Liu S, Li H, Hui N, Wang F and Liu H: Differentiation potential of human embryonic mesenchymal stem cells for skin-related tissue. Br J Dermatol 155: 282-291, 2006.

16. Dai Y, Li J, Li J, Dai G, Mu H, Wu Q, Hu K and Cao Q: Skin epithelial cells in mice from umbilical cord blood mesenchymal stem cells. Burns 33: 418-428, 2007.

17. Gang EJ, Jeong JA, Hong SH, Hwang SH, Kim SW, Yang IH, Ahn C, Han $\mathrm{H}$ and Kim H: Skeletal myogenic differentiation of mesenchymal stem cells isolated from human umbilical cord blood. Stem Cells 22: 617-624, 2004.

18. Thaler R, Rumpler M, Spitzer S, Klaushofer K and Varga F: Mospd1, a new player in mesenchymal versus epidermal cell differentiation. J Cell Physiol 226: 2505-2515, 2011.

19. Deodato B, Arsic N, Zentilin L, Galeano M, Santoro D, Torre V, Altavilla D, Valdembri D, Bussolino F, Squadrito F and Giacca M: Recombinant AAV vector encoding human VEGF165 enhances wound healing. Gene Ther 9: 777-785, 2002.

20. Ha XQ, Lü TD, Hui L and Dong F: Effects of mesenchymal stem cells transfected with human hepatocyte growth factor gene on healing of burn wounds. Chin J Traumatol 13: 349-355, 2010.

21. Liechty KW, Nesbit M, Herlyn M, Radu A, Adzick NS and Crombleholme TM: Adenoviral-mediated overexpression of platelet-derived growth factor-B corrects ischemic impaired wound healing. J Invest Dermatol 113: 375-383, 1999.

22. Cillo C, Cantile M, Faiella A and Boncinelli E: Homeobox genes in normal and malignant cells. J Cell Physiol 188: 161-169, 2001.

23. Ford HL: Homeobox genes: a link between development, cell cycle, and cancer? Cell Biol Int 22: 397-400, 1998.

24. Li J, Li Y, Ma S, Gao Y, Zuo Y and Hu J: Enhancement of bone formation by BMP-7 transduced MSCs on biomimetic nano-hydroxyapatite/polyamide composite scaffolds in repair of mandibular defects. J Biomed Mater Res A 95: 973-981, 2010. 